\title{
On Design of Robust Fault Detection Filter in Finite Frequency Domain with Regional Pole Assignment
}

\author{
Sunjie Zhang, Zidong Wang, Derui Ding, Huisheng Shu, Tasawar Hayat and Abdullah M. Dobaie
}

\begin{abstract}
This paper is concerned with the fault detection filter design problem for uncertain linear discrete-time system in finite-frequency domain with regional pole assignment. An optimized fault detection filter is designed such that 1) the fault detection dynamics is quadratically $D$-stable; 2 ) the effect from the exogenous disturbance on the residual is attenuated with respect to a minimized $H_{\infty}$-norm; and 3 ) the sensitivity of the residual to the fault is enhanced by means of a maximized $H_{-}-$ norm. With the aid of the generalized Kalman-Yakubovich-Popov lemma, the mixed $H_{-} / H_{\infty}$ performance and the $D$-stability requirement are guaranteed by solving a convex optimization problem. An iterative algorithm for designing the desired fault detection filter is proposed by evaluating the threshold on the generated residual function. A simulation result is exploited to illustrate the effectiveness of the proposed design technique.
\end{abstract}

Index Terms-Fault detection filter, regional pole assignment, finite frequency domain, parameter uncertainties, GKYP lemma.

\section{INTRODUCTION}

Over the past few decades, the fault detection (FD) problem has been drawing an increasing research interest, see e.g. [3], [18]. In particular, the $H_{\infty}$ index has been used for evaluating the disturbance attenuation level of the residuals and the $H_{-}$ index has been recognized for measuring the sensitivity to the faults. In [4], the mixed $H_{-} / H_{\infty}$ FD problem has been investigated for Takagi-Sugeno fuzzy model with sensor faults and unknown bounded disturbances. The residual robustness and fault sensitivity conditions have been derived in [15] for the $H_{-} / H_{\infty}$ FD problem via linear matrix inequalities (LMIs). On the other hand, it is well known that the dynamics of a linear system is closely related to the location of its poles [7], [21]. Clearly, for the system governing the FD dynamics, if its poles are assigned within a specified region, then the dynamics of the detection error would have the expected transient

This work was supported in part by the Deanship of Scientific Research (DSR) at King Abdulaziz University in Saudi Arabia under Grant 16-13535-HiCi, the National Natural Science Foundation of China under Grants 61134009 and 61203139, the Royal Society of the U.K., and the Alexander von Humboldt Foundation of Germany.

S. Zhang, D. Ding and H. Shu are with the School of Information Science and Technology, Donghua University, Shanghai 200051, China.

Z. Wang is with the Department of Computer Science, Brunel University, Uxbridge, Middlesex, UB8 3PH, United Kingdom. He is also with the Communication Systems and Networks (CSN) Research Group, Faculty of Engineering, King Abdulaziz University, Jeddah 21589, Saudi Arabia. (Email: Zidong.Wang@brunel.ac.uk)

T. Hayat is with the Department of Mathematics, Quaid-I-Azam University, Islamabad 44000, Pakistan. He is also with the Nonlinear Analysis and Applied Mathematics (NAAM) Research Group, Department of Mathematics, Faculty of Science, King Abdulaziz University, Jeddah 21589, Saudi Arabia

A. M. Dobaie is with the Communication Systems and Networks (CSN) Research Group, Faculty of Engineering, King Abdulaziz University, Jeddah 21589, Saudi Arabia. performance [22]. So far, the mixed $H_{-} / H_{\infty}$ FD problem with regional pole assignment has received little research attention especially for uncertain discrete-time systems.

It is quite common that the faults of a system occur in the finite-frequency range in reality with examples including the incipient fault signal changing slowly which belongs to the low-frequency domain. In [12], [19], the Kalman-YakubovichPopov (KYP) lemma has been employed to establish the equivalence between the frequency domain inequalities of a transfer function and an LMI of its state space realization. Note that the standard KYP lemma treats the frequency domain inequalities for the entire frequency range. Recently, the KYP lemma has been extended in [13] to the finite-frequency band leading to the so-called generalized KYP (GKYP) lemma, where an LMI characterization of the frequency domain inequalities in finite frequency ranges has been provided to facilitate the design of the performance index over the chosen finite or infinite frequency ranges. The FD problem has been thoroughly investigated in [6], [16] in finite frequency domain for networked control systems with or without missing measurements, where the notion of finite frequency stochastic $H_{-}$index has been introduced to measure the sensitivity of the residuals [1], [2], [8], [17]. Recently, in [22], a multi-constrained fault estimation observer with finite frequency specifications has been researched for continuous-time systems.

Up to now, the mixed $H_{-} / H_{\infty}$ fault detection filtering problem for uncertain system has been preliminarily studied, the filter design problems with regional poles placement have gained particular research attention and the GKYP lemma provides an LMI characterization of frequency domain inequalities in the finite-frequency domain. To this end, a seemingly natural research problem arises as follows: in the finite-frequency domain, can we design a mixed $H_{-} / H_{\infty}$ fault detection filter for uncertain linear discrete-time systems with regional pole placement? This filter design issue actually implies four performance indices: 1) the disturbance attenuation requirement through guaranteed $H_{\infty}$ index; 2) the sensitivity requirement to the residuals through guaranteed $H_{-}$ index; 3) the transient behavior of the dynamics of the fault detection error through the regional pole assignment; and 4) the performance robustness against parameter uncertainties. Unfortunately, such a research problem has not gained adequate research attention yet despite its significant engineering practice, and this constitutes our main motivation.

The main contributions of this paper can be highlighted as follows: 1) the mixed $H_{-} / H_{\infty}$ fault detection filtering problem for discrete-time uncertain system is investigated by accommodating the quadratic $D$-stability, the disturbance 
attenuation as well as the sensitivity to faults; 2) a sufficient condition ensuring the quadratic $D$-stability is established in virtue of the projection lemma; and 3) the GKYP lemma is introduced to derive existence conditions for the desired filters.

Notation: For a matrix $M, M^{T}, M^{*}$ and $M^{\perp}$ refer to its transpose, complex conjugate transpose and orthogonal complements, respectively. The Hermitian part of a square matrix $M$ is denoted by $\operatorname{He}(M)=M+M^{*}$. The symbol $\otimes$ denotes the Kronecker product, and $\operatorname{diag}\{\ldots\}$ stands for a block-diagonal matrix.

\section{Problem Formulation AND Preliminaries}

Consider the following uncertain discrete-time system:

$$
\left\{\begin{aligned}
x(k+1) & =(A+\Delta A) x(k)+D_{1} w(k)+G_{1} f(k) \\
y(k) & =C x(k)+D_{2} w(k)+G_{2} f(k) \\
z(k) & =E x(k)
\end{aligned}\right.
$$

where $x(k) \in \mathbb{R}^{n}$ is the state vector, $y(k) \in \mathbb{R}^{r}$ is the measured output, $z(k) \in \mathbb{R}^{s}$ is the output signal to be estimated, $w(k)$ is the noise input which belongs to $L_{2}[0, \infty)$ with known frequency range, and $f(k) \in \mathbb{R}^{l}$ is the fault to be detected. $A, C, D_{1}, D_{2}, E, G_{1}$ and $G_{2}$ are known real matrices with appropriate dimensions. $\triangle A$ is a perturbation matrix representing the parameter uncertainty satisfying $\Delta A=H \Delta B$ where $H$ and $B$ are known constant matrices and $\Delta$ is an unknown matrix subject to $\Delta^{T} \Delta \leq I$. Without loss of generality, we take $x(0)=0$.

Let the fault detection filter be of the following form:

$$
\left\{\begin{aligned}
\hat{x}(k+1) & =A \hat{x}(k)+K(y(k)-C \hat{x}(k)) \\
r(k) & =N(y(k)-C \hat{x}(k)) \\
\hat{z}(k) & =E \hat{x}(k)
\end{aligned}\right.
$$

where $\hat{x}(k) \in \mathbb{R}^{n}$ is the estimated state, $r(k) \in \mathbb{R}^{l}$ is the residual signal, $\hat{z}(k) \in \mathbb{R}^{s}$ is an estimate for $z(k)$, and $K$ and $N$ are the filter parameters to be determined.

Defining $x_{e}(k)=\left[x^{T}(k), \hat{x}^{T}(k)\right]^{T}$, the augmented system from (1) and (2) can be expressed as

$$
\left\{\begin{aligned}
x_{e}(k+1) & =\left(A_{e}+\Delta A_{e}\right) x_{e}(k)+D_{e} w(k)+G_{e} f(k) \\
r(k) & =N\left(C_{e} x_{e}(k)+D_{2} w(k)+G_{2} f(k)\right)
\end{aligned}\right.
$$

where

$$
\begin{aligned}
A_{e} & =\left[\begin{array}{cc}
A & 0 \\
K C & A-K C
\end{array}\right], C_{e}=[C-C], \\
\Delta A_{e} & =\left[\begin{array}{c}
H \\
0
\end{array}\right] \Delta\left[\begin{array}{ll}
B & 0
\end{array}\right]:=H_{e} \Delta B_{e}, \\
D_{e} & =\left[\begin{array}{c}
D_{1} \\
K D_{2}
\end{array}\right], G_{e}=\left[\begin{array}{c}
G_{1} \\
K G_{2}
\end{array}\right] .
\end{aligned}
$$

In this paper, a so-called LMI region is any subset $D$ in the open unit disk described as follows [7], [21]:

$$
D=\left\{\lambda \in \mathbb{C}: f_{D}(\lambda)=L+\lambda M+\bar{\lambda} M^{T}<0\right\},
$$

where $L$ and $M$ are real matrices with $L^{T}=L$. The matrixvalued function $f_{D}(\lambda)$ is called the characteristic function of $D$. As explained in [21], with different choices of the matrices
$L$ and $M$, the LMI region $D$ defined in (4) can be used to represent many kinds of popular pole regions, such as disk, vertical strips, horizontal strips, conic sector, etc.

Definition 1. [7] The system $x(k+1)=A x(k)$ is said to be $D$-stable if all its poles (eigenvalues of $A$ ) lie in $D$.

Definition 2. [7] System (3) is said to be quadratically Dstable if there exists a symmetric positive definite matrix $X$ such that the following matrix inequality

$$
L \otimes X+M \otimes\left(X\left(A_{e}+\Delta A_{e}\right)\right)+M^{T} \otimes\left(\left(A_{e}+\Delta A_{e}\right)^{T} X\right)<0
$$

is true where the LMI region $D$ is defined in (4).

Remark 1. It has been revealed in [7] that, if (5) is satisfied, then all poles of the uncertain time-invariant matrix $A_{e}+\Delta A_{e}$ are constrained to lie within the specified LMI region $D$.

For the plant model (1) and the fault detection filter (2) with a given LMI region $D$, our objective in this paper is to design the filter parameters $K$ and $N$ such that the following three requirements are simultaneously satisfied in the presence of the parameter uncertainties:

(i) All poles of the augmented system (3) are constrained to lie inside a prescribed LMI region D, that is, (3) is quadratically $D$-stable.

(ii) Under the zero-initial condition and $f(k)=0$, the transfer function $G_{r w}\left(e^{j \theta}\right)=N C_{e}\left(e^{j \theta} I-A_{e}-\Delta A_{e}\right)^{-1} D_{e}+N D_{2}$ from the noise input $w(k)$ to the residual $r(k)$ satisfies the constraint [14]

$$
\left\|G_{r w}\left(e^{j \theta}\right)\right\|_{\infty}:=\sup \left\{\sigma_{\max }\left(G_{r w}\left(e^{j \theta}\right)\right)\right\}<\gamma_{w}, \forall|\theta| \leq \theta_{l}
$$

where $\sigma_{\max }(\cdot)$ denotes the largest singular value of $(\cdot)$, and $\gamma_{w}>0$ is made as small as possible in the feasibility of (6) so as to minimize the effect from the exogenous disturbance on the residual.

(iii) Under the zero-initial condition and $w(k)=0$, the transfer function $G_{r f}\left(e^{j \theta}\right)=N C_{e}\left(e^{j \theta} I-A_{f}-\Delta A_{e}\right)^{-1} G_{e}+$ $N G_{2}$ from the fault $f(k)$ to the residual $r(k)$ satisfies the constraint [15]

$$
\left\|G_{r f}\left(e^{j \theta}\right)\right\|_{-}:=\inf \left\{\sigma_{\min }\left(G_{r f}\left(e^{j \theta}\right)\right)\right\}>\gamma_{f}, \quad \forall|\theta| \leq \theta_{l}
$$

where $\sigma_{\min }(\cdot)$ denotes the smallest singular value of $(\cdot)$, and $\gamma_{f}>0$ is made as large as possible in the feasibility of (7) so as to enhance the sensitivity of faults on the residual.

We further adopt a residual evaluation stage including an evaluation function $J(L)$ and a threshold $J_{t h}$ of the following form: $J(L):=\left\{\sum_{k=0}^{L} r^{T}(k) r(k)\right\}^{\frac{1}{2}}$ and $J_{t h}:=$ $\sup _{f=0} J(L)$ where $L$ denotes the length of the finite evaluating time-horizon. The fault is detected by using the following logical relation: $J(L)>J_{t h} \Longrightarrow$ the fault is detected $\Longrightarrow$ alarm, $J(L) \leq J_{t h} \Longrightarrow$ no fault.

Remark 2. The addressed filter design problem is actually a multiobjective optimization one that can be achieved by designing the threshold $J_{t h}$ subject to $\min J_{t h}=\frac{\min \gamma_{w}}{\max \gamma_{f}}$. 


\section{Main Results}

Lemma 1. (Projection Lemma) [11] Given a symmetric matrix $\Psi$ and two matrices $\Gamma$ and $\Lambda$, the following inequality $\Psi+\Gamma \Phi \Lambda^{T}+\Lambda \Phi^{T} \Gamma^{T}<0$ is solvable with respect to the decision matrix $\Phi$ if and only if $\Gamma^{\perp} \Psi \Gamma^{\perp T}<0, \Lambda^{\perp} \Psi \Lambda^{\perp T}<0$.

Lemma 2. (S-procedure) Let $L=L^{T}, H, \Delta$ and $B$ be real matrices with $\Delta$ satisfying $\Delta^{T} \Delta \leq I$. Then, $L+H \Delta B+$ $B^{T} \Delta^{T} H^{T}<0$ if and only if there exists a positive scalar $\varepsilon>0$ such that $L+\varepsilon^{-1} H H^{T}+\varepsilon B^{T} B<0$.

\section{A. Mixed $H_{-} / H_{\infty}$ Performance}

The Generalized KYP Lemma provides a necessary and sufficient condition in terms of an LMI to ensure that the given transfer function $G\left(e^{j \theta}\right)$ satisfies a desired frequency domain property condition. For convenience, we assume that $\Delta A_{e}=0$.

Lemma 3. Let $\gamma_{w}>0, \gamma_{f}>0, \Pi_{w}=\operatorname{diag}\left\{I,-\gamma_{w}^{2} I\right\} \in$ $\mathbb{R}^{(n+t) \times(n+t)}, \Pi_{f}=\operatorname{diag}\left\{-I, \gamma_{f}^{2} I\right\} \in \mathbb{R}^{(n+t) \times(n+t)}$, a positive scalar $\theta_{f}$ and the filter gains $K, N$ be given. Consider the detection error dynamics (3) with transfer functions $G_{r w}\left(e^{j \theta}\right)$ and $G_{r f}\left(e^{j \theta}\right)$. The following statements are equivalent:

(a) $\left\|G_{r w}\left(e^{j \theta}\right)\right\|_{\infty}<\gamma_{w}$ and $\left\|G_{r f}\left(e^{j \theta}\right)\right\|_{-}>\gamma_{f}, \forall|\theta| \leq \theta_{l}$.

(b) There exist Hermitian matrices $Q_{1}, Q_{2}>0, Q_{3}$ and $Q_{4}>0$ such that

$$
\begin{aligned}
& {\left[\begin{array}{cc}
A_{e} & D_{e} \\
I & 0
\end{array}\right]^{T} \Xi_{w}\left[\begin{array}{cc}
A_{e} & D_{e} \\
I & 0
\end{array}\right] } \\
+ & {\left[\begin{array}{cc}
N C_{e} & N D_{2} \\
0 & I
\end{array}\right]^{T} \Pi_{w}\left[\begin{array}{cc}
N C_{e} & N D_{2} \\
0 & I
\end{array}\right]<0, } \\
& {\left[\begin{array}{cc}
A_{e} & G_{e} \\
I & 0
\end{array}\right]^{T} \Xi_{f}\left[\begin{array}{cc}
A_{e} & G_{e} \\
I & 0
\end{array}\right] } \\
+ & {\left[\begin{array}{cc}
N C_{e} & N G_{2} \\
0 & I
\end{array}\right]^{T} \Pi_{f}\left[\begin{array}{cc}
N C_{e} & N G_{2} \\
0 & I
\end{array}\right]<0, }
\end{aligned}
$$

where

$$
\begin{aligned}
& \Xi_{w}=\left[\begin{array}{cc}
-Q_{1} & Q_{2} \\
Q_{2} & Q_{1}-\left(2 \cos \theta_{l}\right) Q_{2}
\end{array}\right], \\
& \Xi_{f}=\left[\begin{array}{cc}
-Q_{3} & Q_{4} \\
Q_{4} & Q_{3}-\left(2 \cos \theta_{l}\right) Q_{4}
\end{array}\right] .
\end{aligned}
$$

(c) There exist Hermitian matrices $Q_{1}, Q_{2}>0, Q_{3}, Q_{4}>0$ and general matrix $F$ such that

$$
\begin{aligned}
& {\left[\begin{array}{cc}
-Q_{1} & Q_{2}-F \\
* & Q_{1}-\left(2 \cos \theta_{l}\right) Q_{2}+\operatorname{He}\left(F^{T} A_{e}\right)+C_{e}^{T} \bar{N} C_{e} \\
* & *
\end{array}\right.} \\
& \left.\begin{array}{c}
0 \\
F^{T} D_{e}+C_{e}^{T} \bar{N} D_{2} \\
-\gamma_{w}^{2} I+D_{2}^{T} \bar{N} D_{2}
\end{array}\right]:=\bar{\Theta}_{1}<0, \\
& {\left[\begin{array}{cc}
-Q_{3} & Q_{4}-F \\
* & Q_{3}-\left(2 \cos \theta_{l}\right) Q_{4}+\operatorname{He}\left(F^{T} A_{e}\right)-C_{e}^{T} \bar{N} C_{e} \\
* & *
\end{array}\right.} \\
& \left.\begin{array}{c}
0 \\
F^{T} G_{e}-C_{e}^{T} \bar{N} G_{2} \\
-G_{2}^{T} \bar{N} G_{2}+\gamma_{f}^{2} I
\end{array}\right]:=\bar{\Theta}_{2}<0
\end{aligned}
$$

hold, where $\bar{N}=N^{T} N$.

Proof. This lemma can be proved along the similar line of that for Theorem 1 in [6] by using Projection lemma (Lemma 1) and the Generalized KYP Lemma [13].

\section{B. D-stability}

The mixed $H_{-} / H_{\infty}$ fault detection filter design problem in Lemma 3 is actually concerned with the steady-state and disturbance attenuation behaviors. The transient (dynamical) behavior of the detection error dynamics, on the other hand, is equally important to ensure a fast detection process and therefore it would make practical sense to guarantee the desired quadratic $D$-stability for (3).

Theorem 1. Assume that there exist a symmetric positive definite matrix $P_{s}>0$ and a matrix $F$ such that

$$
\begin{gathered}
\bar{\Theta}_{3}:=\left[\begin{array}{c}
-I \otimes\left(F+F^{T}\right) \\
I \otimes P_{s}-I \otimes F^{T}+M^{T} \otimes\left(A_{e}^{T} F\right) \\
I \otimes P_{s}-I \otimes F+M \otimes\left(F^{T} A_{e}\right) \\
M^{T} \otimes\left(A_{e}^{T} F\right)+M \otimes\left(F^{T} A_{e}\right)+L \otimes P_{s}
\end{array}\right]<0
\end{gathered}
$$

holds. Then, the filtering error dynamics in (3) is quadratically D-stable.

Proof. First, by the properties of the Kronecker product, we can rewrite (12) as

$$
\begin{aligned}
& {\left[\quad-I \otimes\left(F+F^{T}\right)\right.} \\
& I \otimes P_{s}-I \otimes F^{T}+\left(M \otimes A_{e}\right)^{T}(I \otimes F) \\
& I \otimes P_{s}-I \otimes F+\left(I \otimes F^{T}\right)\left(M \otimes A_{e}\right) \\
& \left(M \otimes A_{e}\right)^{T}(I \otimes F)+\left(I \otimes F^{T}\right)\left(M \otimes A_{e}\right)+L \otimes P_{s} \\
& <0
\end{aligned}
$$

Performing simple algebra operations, inequality (13) is equivalent to

$$
\begin{gathered}
{\left[\begin{array}{cc}
0 & I \otimes P_{s} \\
I \otimes P_{s} & L \otimes P_{s}
\end{array}\right]+\left[\begin{array}{c}
-I \\
\left(M \otimes A_{e}\right)^{T}
\end{array}\right](I \otimes F)} \\
\times\left[\begin{array}{ll}
I & I
\end{array}\right]+\left(\left[\begin{array}{c}
-I \\
\left(M \otimes A_{e}\right)^{T}
\end{array}\right](I \otimes F)\left[\begin{array}{ll}
I & I
\end{array}\right]\right)^{T} \\
<0 .
\end{gathered}
$$

Next, it is easy to check that the null space of $\left[-I,\left(M \otimes A_{e}\right)\right]^{T}$ is $\left[\left(M \otimes A_{e}\right)^{T}, I\right]$ and the null space of $[I, I]^{T}$ is $[-I, I]$. Note that

$$
\begin{gathered}
\Omega_{1}:=L \otimes P_{s}+M \otimes\left(P_{s} A_{e}\right)+M^{T} \otimes\left(A_{e}^{T} P_{s}\right) \\
\Omega_{2}:=(L-2 I) \otimes P_{s} .
\end{gathered}
$$

By Lemma 1, (14) holds if and only if $\Omega_{1}<0$ and $\Omega_{2}<0$. It follows from Definition 1 that the system (3) is quadratically $D$-stable.

Remark 3. The D-stability issue has been investigated in [1], [2], [5], [8], [17], where the projection lemma has been utilized. In this paper, by using the same method combined with the Kronecker product approach, the proof is preceded in a more compact way. In comparison with the result in [7], the obtained sufficient condition is more suitable for designing 
a stable fault detection filter in the finite frequency domain. On the other hand, it follows from [1], [2], [13] that both Lemma 2 and GKYP Lemma can be interpreted as special cases of a generalized version of the $S$-procedure. In this paper, for the benefits of engineering applications, the $H_{-}$index, $H_{\infty}$ index, $D$-stability and robustness have been introduced as individual performance constraints on the in order to facilitate the realtime fault detection implementation.

\section{Fault detection filter design}

Having obtained the analysis results, we are now in a position to solve the fault detection filter design problem. It can be seen from Lemma 3 that the inequality (10) is still non-convex due to the product term between the filter gain matrix $K$ and the slack variable $F$. To overcome such a coupling problem, we set $F=\left[\begin{array}{cc}F_{1} & F_{2} \\ F_{3} & F_{3}\end{array}\right], G=K^{T} F_{3}$ and then have $F^{T} A_{e}=\left[\begin{array}{cc}F_{1}^{T} A+G^{T} C & F_{3}^{T} A-G^{T} C \\ F_{2}^{T} A+G^{T} C & F_{3}^{T} A-G^{T} C\end{array}\right]:=$ $\mathcal{A}, F^{T} D_{e}=\left[D_{1}^{T} F_{1}+D_{2}^{T} G, D_{1}^{T} F_{2}+D_{2}^{T} G\right]^{T}:=\mathcal{D}, F^{T} G_{e}=$ $\left[G_{1}^{T} F_{1}+G_{2}^{T} G, G_{1}^{T} F_{2}+G_{2}^{T} G\right]^{T}:=\mathcal{G}$.

Next, the results obtained for the nominal dynamic will be extended to uncertain augmented system described in (3) with $\Delta A_{e} \neq 0$. Following Lemma 3 and Theorem 1 and summarizing the discussions made so far, we are now ready to present our main results.

Theorem 2. Let $\gamma_{w}>0$ and $\gamma_{f}>0$ be given. Assume that there exist positive definite matrices $P_{s}>0, Q_{2}>0, Q_{4}>0$, matrices $F, W_{1}, W_{2}, Q_{1}, Q_{3}, \bar{N}$ and positive constant scalars $\varepsilon_{i}>0(i=1,2,3)$ such that the following inequalities hold:

$$
\left[\begin{array}{ccc}
\Theta_{i} & H_{i} & \varepsilon_{i} B_{i}^{T} \\
H_{i}^{T} & -\varepsilon_{i} I & 0 \\
\varepsilon_{i} B_{i} & 0 & -\varepsilon_{i} I
\end{array}\right]<0, \quad i=1,2,3 .
$$

where

$$
\begin{aligned}
& \Theta_{1}=\left[\begin{array}{cc}
-Q_{1} & Q_{2}-F \\
* & Q_{1}-\left(2 \cos \theta_{l}\right) Q_{2}+\operatorname{He}(\mathcal{A})+C_{e}^{T} \bar{N} C_{e} \\
* & *
\end{array}\right. \\
& \left.\begin{array}{c}
0 \\
\mathcal{D}+C_{e}^{T} \bar{N} D_{2} \\
-\gamma_{w}^{2} I+D_{2}^{T} \bar{N} D_{2}
\end{array}\right] \\
& \Theta_{2}=\left[\begin{array}{cc}
-Q_{3} & Q_{4}-F \\
* & Q_{3}-\left(2 \cos \theta_{l}\right) Q_{4}+\operatorname{He}(\mathcal{A})-C_{e}^{T} \bar{N} C_{e} \\
* & *
\end{array}\right. \\
& \left.\begin{array}{c}
0 \\
\mathcal{G}-C_{e}^{T} \bar{N} G_{2} \\
-G_{2}^{T} \bar{N} G_{2}+\gamma_{f}^{2} I
\end{array}\right] \\
& \Theta_{3}=\left[\begin{array}{c}
-I \otimes\left(F+F^{T}\right) \\
I \otimes P_{s}-I \otimes F^{T}+M^{T} \otimes \mathcal{A}^{T}
\end{array}\right. \\
& I \otimes P_{s}-I \otimes F+M \otimes \mathcal{A} \\
& \left.M^{T} \otimes \mathcal{A}^{T}+M \otimes \mathcal{A}+L \otimes P_{s}\right], \\
& H_{1}=H_{2}=\left[\begin{array}{lll}
0 & H_{e}^{T} F & 0
\end{array}\right]^{T}, B_{1}=B_{2}\left[\begin{array}{lll}
0 & B_{e} & 0
\end{array}\right] \text {, } \\
& H_{3}=\left[\begin{array}{l}
M_{1}^{T} \otimes\left(F^{T} H_{e}\right) \\
M_{1}^{T} \otimes\left(F^{T} H_{e}\right)
\end{array}\right], B_{3}=\left[\begin{array}{ll}
0 & M_{2} \otimes\left(B_{e}\right)
\end{array}\right] .
\end{aligned}
$$

In this case, the uncertain filtering error dynamics (3) is quadratically $D$-stable and satisfies the $H_{\infty}$ performance index (6) as well as the $H_{-}$performance index (7) in finite frequency domain. Furthermore, if $\left(P_{s}, Q_{1}, Q_{2}, Q_{3}, Q_{4}, F, G, \bar{N}\right)$ is a feasible solution of (17), then the fault detection filter parameters $K$ and $N$ can be obtained by means of the matrices $F, G$ and $\bar{N}$, respectively, where $N$ is a factorization of $\bar{N}$ (i.e., $\bar{N}=N^{T} N$ ) and $K=F_{3}^{-T} G^{T}$.

Proof. Factorize the matrix $M$ as $M=M_{1}^{T} M_{2}$, where $M_{1}$ and $M_{2}$ have full column rank. Such a factorization can be obtained easily through the singular value decomposition (SVD) technique.

Replace $A_{e}$ in (10), (11) and (12) with $A_{e}+H_{e} \Delta B_{e}$, and rewrite (10), (11) and (12) in the following form:

$$
\bar{\Theta}_{i}+H_{i} \Delta B_{i}+B_{i}^{T} \Delta^{T} H_{i}^{T}<0, i=1,2,3 .
$$

Applying Lemma 2 to (18), respectively, we obtain the following inequalities

$$
\left[\begin{array}{ccc}
\bar{\Theta}_{i} & H_{i} & \varepsilon_{i} B_{i}^{T} \\
H_{i}^{T} & -\varepsilon_{i} I & 0 \\
\varepsilon_{i} B_{i} & 0 & -\varepsilon_{i} I
\end{array}\right]<0, i=1,2,3 .
$$

on the positive definite matrix $P_{s}>0$ and the positive scalar parameters $\varepsilon_{i}>0$. After tedious algebraic manipulations, (19) can be transformed into (17) which can be handled directly by the LMI optimization technique. The details of the proof are omitted here for brevity.

Remark 4. For the addressed fault detection filter design problem, in order to maximize the sensitivity to faults and minimize the influence from disturbances, a sub-optimal filter gain matrix $K$ can be determined by $K=F_{3}^{-T} G^{T}$ via solving the following optimization problem:

$$
\min _{Q_{2}>0, Q_{4}>0, P_{s}>0, F, G, Q_{1}, Q_{3}, \bar{N}} J_{t h} \text { subject to (17). }
$$

So far, we have designed the fault detection filter and it remains to evaluate the generated residual. For this purpose, we propose an iterative algorithm for the evaluation of the threshold $J_{t h}$ for the fault detection filter in finite frequency.

The fault detection filter design (FDFD) algorithm:

Step 1. Solve (17) (where $i=3$ ) for $P_{s}>0, F$ and $G$. Calculate $K_{0}$ by $K_{0}=F_{3}^{-T} G^{T}$. Let $K=K_{0}$.

Step 2. With $K$ from Step 1, obtain $\min \gamma_{w}$ and $\max \gamma_{f}$ by solving (17) (where $i=1,2$ ), respectively.

Step 3. If, with $\gamma_{w}$ and $\gamma_{f}$ replaced by $\min \gamma_{w}$ and $\max \gamma_{f}$ respectively, (17) (where $i=1,2$ ) are feasible for Theorem 2 , then the locally optimized parameters $K$ and $N$ can be obtained and the job is done. Otherwise, go to Step 4.

Step 4. Decrease $\min \gamma_{w}$ by $\mu$ and increase $\max \gamma_{f}$ by $\mu$ where $\mu>0$ is a sufficiently small scalar. Solve (17) with the updated $\min \gamma_{w}$ and $\max \gamma_{f}$. Repeat such a procedure until $\left|J_{t h}(i)-J_{t h}(i-1)\right|<\delta(i$ is the iteration number). In the feasibility of (17), the locally optimized filter parameters $K$ and $N$ as well as the index $\min J_{t h}=\min \gamma_{w} / \max \gamma_{f}$ can be obtained.

Step 5. Stop. 
Remark 5. It is obvious that, the key parameters min $\gamma_{w}$ and $\max \gamma_{f}$ can be, respectively, obtained in Step 3 and Step 4 by fixing $K$. Such a strategy can guarantee the iterative result to be always better than that obtained in last iteration or, at least, the same. In this case, the value of $J_{t h}$ is monotonically decreasing as long as the iterative process is carried out continuously. In the iterative process, the stability constraint (17) (where $i=3$ ) is always required to be satisfied, which means that the fault detection observer is quadratically $D$ stable. Nevertheless, the FDFD algorithm cannot guarantee the optimality of the addressed multiobjective problem. Also, with the LMI solvers, the iterative process might provide oscillations around suboptimal values and does not really converge if certain accuracy is required. These algorithmic issues deserve further investigations in our future research.

\section{ILLUSTRATIVE EXAMPLE}

Consider the system (1) with

$$
\begin{aligned}
& A=\left[\begin{array}{cc}
-0.4830 & -0.3478 \\
0.4678 & 0.5242
\end{array}\right], D_{1}=\left[\begin{array}{c}
-0.6268 \\
0.4663
\end{array}\right], \\
& G_{1}=\left[\begin{array}{ll}
-0.6628 & 0.7272
\end{array}\right]^{T}, C=\left[\begin{array}{ll}
0.3946 & -0.544
\end{array}\right], \\
& E=\left[\begin{array}{ll}
1 & 0.5
\end{array}\right], D_{2}=0.1724, G_{2}=0.4447 \\
& H=[0.01,0,0.02]^{T}, \Delta=0.4, B=[0.01,0,0.03] .
\end{aligned}
$$

The poles are restricted in the intersection of the disk centered at $(-\alpha, 0)$ with radius $r$ and the vertical strip $\operatorname{Re}(\lambda)<-\alpha_{1}$, where $\alpha=0, r=0.8$ and $\alpha_{1}=-0.5$. Such a pole region can be described by $D=D_{1} \cap D_{2}$, $D_{i}=\left\{\lambda \in \mathbb{C}: f_{D_{i}}(\lambda)=L_{i}+\lambda M_{i}+\bar{\lambda} M_{i}^{T}<0\right\}$, $M_{i}=M_{i 1}^{T} M_{i 2}(i=1,2)$ where $L_{1}=\left[\begin{array}{cc}-r & \alpha \\ \alpha & -r\end{array}\right], M_{1}=$ $\operatorname{diag}\{0,1\}, M_{11}=\left[\begin{array}{ll}1 & 0\end{array}\right], M_{22}=\left[\begin{array}{ll}0 & 1\end{array}\right], L_{2}=2 \alpha_{1}, M_{2}=$ $1, M_{21}=1, M_{22}=1$.

For the initial value $x(0)=\hat{x}(0)=[0,0,0]^{T}$, the exogenous disturbance input is set as $w(k)=\frac{1}{k} \sin (k)$. The frequency domain is $|\theta| \leq \theta_{l}$ with $\theta_{l}=0.3$. The fault signal $f(k)$ is given as follow: $f(k)=\left\{\begin{array}{cc}0.75 & 100<k<200 \\ 0 & \text { elsewhere. }\end{array}\right.$ In our iterative algorithm, we set $\mu=0.01$ and $\delta=0.05$. The corresponding filter gain matrices are obtained as $K=[0.12401,-0.11083]^{T}$ and $N=236.43$. Fig. 1 plots the generated residual signal. The simulation result confirms the effectiveness of the proposed algorithm.

\section{REFERENCES}

[1] O. Bachelier and D. Mehdi, Robust matrix root-clustering through extended KYP lemma, SIAM Journal of Control and Optimization, vol. 45, no. 1, pp. 368-381, Jul. 2006.

[2] J. Bosche, O. Bachelier, and D. Mehdi, An approach for robust matrix root-clustering analysis in a union of regions, IMA Journal of Mathematical Control and Information, vol. 22, no, 3, pp. 227-239, Sept. 2005.

[3] K. Bouibed, L. Seddiki, K. Guelton and H. Akdag, Actuator and sensor fault detection and isolation of an actuated seat via nonlinear multiobservers, Systems Science and Control Engineering: An Open Access Journal, vol. 2, no. 1, pp. 150-160, 2014.

[4] M. Chadli, A. Abdo and S. X. Ding, $H_{-} / H_{\infty}$ fault detection filter design for discrete-time Takagi-Sugeno fuzzy system, Automatica, vol. 49, no. 7, pp. 1996-2005, Jul. 2013.

[5] X. Chang and G. Yang, Non-fragile fuzzy $H_{\infty}$ filter design for nonlinear continuous-time systems with $D$ stability constraints, Signal Processing, vol. 92, no. 2, pp. 575-586, Feb. 2012.

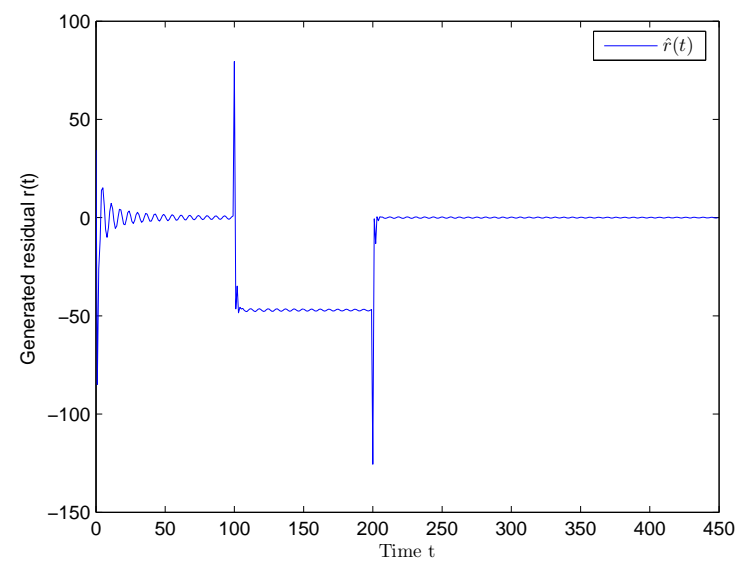

Fig. 1. the generated residual signal of $r(k)$.

[6] J. Chen and Y. Cao, A stable fault detection observer design in finite frequency domain, International Journal of Control, vol. 86, no. 2 , pp. 290-298, Feb. 2013.

[7] M. Chilali and P. Gahinet, $H_{\infty}$ design with pole placement constraints: an LMI approach. IEEE Transactions on Automatic Control, vol. 41, no. 3 pp. 358-367, Mar. 1996.

[8] M. C. de Oliveira, J. Bernussou, and J. C. Geromel, A new discretetime robust stability condition, Systems \& Control Letters, vol. 37, no. 4, Jul. 1999.

[9] D. Ding, X. Li and Y. Wang, Nonfragile filtering for discrete-time linear systems in finite-frequency domain, International Journal of Control, vol. 86, no. 4, pp. 664-673, Apr. 2013

[10] H. Dong, Z. Wang and H. Gao, Fault detection for Markovian jump systems with sensor saturations and randomly varying nonlinearities, IEEE Transactions on Circuits and Systems II: Regular Papers, vol. 59, no. 10, pp. 2354-2362, Oct. 2012.

[11] P. Gahinet and P. Apkarian, A linear matrix inequality approach to $H_{\infty}$ control, International Journal of Robust Nonlinear Control, vol. 4, no. 4 , pp. 421-448, Mar. 1994.

[12] B. Hencey and A. G. Alleyne, A KYP lemma for LMI regions, IEEE Trans. Automatic Control, vol. 52, no. 10, pp. 1926-1930, Oct. 2007.

[13] T. Iwasaki and S. Hara, Generalized KYP lemma: unified frequency domain inequalities with design applications, IEEE Trans. Automatic Control, vol. 50, no. 1, pp. 41-59, Jan. 2005.

[14] X. Li and G.-H. Yang, Fault estimation for discrete-time delay systems in finite frequency domain, In: Proc. 2009 American Control Conference, pp. 4328-4333, Louis, MO, USA, Jun. 2009.

[15] J. Liu, J. L. Wang and G.-H. Yang, An LMI approach to minimum sensitivity analysis with application to fault detection, Automatica, vol. 41, no. 11, pp. 1995-2004, Nov. 2005.

[16] Y. Long and G.-H. Yang, Fault detection in finite frequency domain for networked control systems with missing measurements, Journal of the Franklin Institute, vol. 350, no. 9, pp. 2605-2626, Nov. 2013

[17] D. Peaucelle, D. Arzelier, O. Bachelier, and J. Bernussou, A new robust D-stability conditionfor real convex polytopic uncertainty, Systems \& Control Letters, vol. 40, no. 1 pp. 21-30, May. 2000.

[18] L. Qin, X. He and D. Zhou, A survey of fault diagnosis for swarm systems, Systems Science and Control Engineering: An Open Access Journal, vol. 2, no. 1, pp. 13-23, 2014.

[19] A. Rantzer, On the Kalman-Yakubovich-Popov lemma, Systems \& Control Letters, vol. 28, no 1, pp. 7-10, Jun. 1996.

[20] B. Shen, S. X. Ding and Z. Wang, Finite-horizon $H_{\infty}$ fault estimation for uncertain linear discrete time-varying systems with known inputs, IEEE Transactions on Circuits and Systems II: Express Briefs, vol. 60, no. 12, pp. 902-906, Dec. 2013.

[21] F. Yang, Z. Wang, and H. Shu, Mixed $H_{2} / H_{\infty}$ filtering for uncertain systems with regional pole assignment, IEEE Trans. Aerospace and Electronic System, vol. 41, no. 2, pp. 438-448, Apr. 2005.

[22] K. Zhang, B. Jiang, P. Shi and J. Xu, Multi-constrained fault estimation observer design with finite frequency specifications for continuous-time systems, International Journal of Control, vol. 87, no. 8, pp.1635-1645, Feb. 2014. 